\title{
Is headache related with Vitamin D insufficiency?
}

\author{
Yi Yang $\cdot$ Hong-Liang Zhang $\cdot$ Jiang Wu
}

Received: 7 June 2010/ Accepted: 20 June 2010/Published online: 3 July 2010

(C) Springer-Verlag 2010

Sir,

In the article by Prakash et al. [1], a relation between prevalence of headache and the latitude was reviewed. Although the profile of headache matches with seasonal variations and geographical distribution of serum vitamin D levels, we believe that it is currently far-fetched to correlate headache with vitamin D insufficiency.

Several lines of epidemiological and experimental evidence have been proposed to support links of vitamin D with various conditions or diseases beyond bone health, including muscle function, autoimmune diseases, cancer, cardiovascular diseases (CVD), hypertension, diabetes, inflammatory diseases, all-cause mortality, etc. [2].

The relations between vitamin D insufficiency and human diseases, especially multiple sclerosis has been partly explained by the seasonal and geographical variations of serum vitamin $\mathrm{D}$, which has further been confirmed by decreased incidence and severity of multiple sclerosis by vitamin $\mathrm{D}$ supplementation [3]. In this regard, either observational studies to find decreased levels of serum vitamin D in patients with headache or placebo-controlled studies to observe the therapeutic effects of vitamin D in headache are still lacking.

The geographical features of apolipoprotein $\mathrm{E}(\mathrm{apoE})$ allele [4] and serum vitamin D concentrations [5], both of

Y. Yang · H.-L. Zhang · J. Wu

Department of Neurology, First Hospital of Jilin University, Changchun, China

\section{H.-L. Zhang ( $\square)$}

Department of Neurobiology, Care Sciences and Society,

NVS, Karolinska Institute, Novum, plan 5,

14186 Stockholm, Sweden

e-mail: Hongliang.Zhang@ki.se which are associated with autoimmune diseases such as multiple sclerosis, also lead us to assume that there might be some correlations between apoE epsilon 4 allele and vitamin D. However, since robust evidence is lacking, from the current knowledge, we can only ascribe this to coincidence, unless new evidence can be found in future studies. Otherwise wearing more clothes may also be linked to the higher incidence of stoke in cold areas.

In summary, it is promising to confirm a positive correlation between headache and vitamin $\mathrm{D}$ insufficiency in that it may provide new therapeutic strategies for migraine as well as other types of headaches. However, further investigations are still needed to find out such a relation between headache and vitamin $\mathrm{D}$.

Conflict of interest None.

\section{References}

1. Prakash S, Mehta NC, Dabhi AS et al (2010) The prevalence of headache may be related with the latitude: a possible role of Vitamin D insufficiency? J Headache Pain (epub ahead of print)

2. Correale J, Ysrraelit MC, Gaitán MI (2009) Immunomodulatory effects of Vitamin D in multiple sclerosis. Brain 132(Pt 5):11461160

3. Cantorna MT, Mahon BD (2004) Mounting evidence for vitamin D as an environmental factor affecting autoimmune disease prevalence. Exp Biol Med 229(11):1136-1142

4. Gerdes LU (2003) The common polymorphism of apolipoprotein E: Geographical aspects and new pathophysiological relations. Clin Chem Lab Med 41(5):628-631

5. Beretich BD, Beretich TM (2009) Explaining multiple sclerosis prevalence by ultraviolet exposure: a geospatial analysis. Mult Scler 15(8):891-898 